\title{
FLUORESCENCE DYNAMICS AND CROSS RELAXATION OF 4+ CURIUM IN CERIUM TETRAFLUORIDE
}

\author{
G. K. Liu and James V. Beitz
Argonne National Laboratory \\ G. K. Liu and James V. Beitz
Argonne National Laboratory \\ Argonne, IL 60439
}

\begin{abstract}
Fluorescence from $\mathrm{Cm}^{4+}$ has been observed for the first time. The dynamics of its emitting $5 f$ state have been probed using time- and frequency-resolved laser-induced fluorescence. Nonexponential fluorescence decays were found from $\mathrm{Cm}_{0.001} \mathrm{Ce}_{0.999 \mathrm{~F}_{4}}$ and $\mathrm{Cm}_{0.05} \mathrm{Ce}_{0.95} \mathrm{~F}_{4}$ at temperatures from $4.2 \mathrm{~K}$ to $300 \mathrm{~K}$. A simple cross-relaxation model was found to fit the excited f-state dynamics of the Cm0.001 Ce0.999F4 sample at low excitation intensity whereas an exciton-exciton annihilation model was necessary to model the observed fluorescence decay as exciton number density increased at higher excitation intensity. The annihilation model, modified to account for a small amount of trap site emission, was required to fit all the observed decays from the $\mathrm{Cm} 0.05 \mathrm{Ce} 0.95 \mathrm{~F}_{4}$ sample.
\end{abstract}

\section{DISCLAIMER}

\begin{abstract}
This report was prepared as an account of work sponsored by an agency of the United States Government. Neither the United States Government nor any agency thereof, nor any of their employees, makes any warranty, express or implied, or assumes any legal ljabjlity or responsibility for the accuracy, completeness, or usefulness of any information, apparatus, product, or process disclosed, or represents that its use would not infringe privately owned rights. Reference herein to any specific commercial product, process, or service by trade name, trademark, manufacturer, or otherwise does not necessarily constitute or imply its endorsement, recommendation, or favoring by the United States Government or any agency thereof. The views and opinions of authors expressed herein do not necessarily state or reflect those of the United States Government or any agency thercof.
\end{abstract}




\section{INTRODUCTION}

Time- and frequency-resolved fluorescence is a particularly useful probe for dynamics of excited states and energy transfer processes associated with nonexponential fluorescence decay and spectral migration of optically excited ions in solids[1,2]. For different materials, the mechanisms responsible for energy transfer can be different. Extensive work has been carried out on transition metal and lanthanide activated materials [3-9], but few actinide studies have been reported.

Stronger coupling between actinide f-states and lattice vibrations than is the case in lanthanide materials $[10,11]$, and larger ion-ion interaction result from the greater radial extent of $5 f$ electron wave functions in comparison with the shielding $6 s$ and $6 \mathrm{p}$ shells. Increased spin-orbital coupling and reduced electronic repulsion of $5 f$ electrons[10,11] result in higher state densities due to more closely spaced Jmultiplets with larger splitting within each multiplet. These factors, together with increased f-f oscillator strengths, are likely to result in enhancement of energy transfer processes such as crossrelaxation and energy up-conversion processes.

In this paper, we report observation of nonexponential fluorescence decays from a $5 f$ state of $\mathrm{Cm}^{4+}$ in

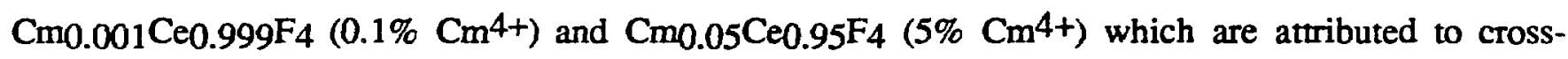
relaxation, exciton-exciton annihilation, and trapping via defect sites. The decay data for $0.1 \% \mathrm{Cm} 4+$ at low excitation intensity are consistent with a cross-relaxtion model, but an exciton-exciton annihilation model is required to fit the higher excitation intensity data as well as all the observed fluorescence decays from the $5 \% \mathrm{Cm}^{4+}$ sample.

\section{EXPERIMENTAL APPARATUS AND SAMPLE PREPARATION}

A tunable dye laser pumped by a Q-switched Nd:YAG laser was used as the excitation source. Fluorescence from the sample contained in a variable temperature cryostat was selected using long pass filters, dispersed with a 1 meter monochromator, and detected using a cooled photomultipliers (GaAs and S-1 photocathodes). Boxcar detectors, whose outputs were digitized and stored on a computer, were 
used to acquire excitation and emission spectra which were normalized to the dye laser energy using a pyroelectric detector. Excitation spectra were calibrated using the optogalvanic effect in a uranium hollow cathode discharge and an etalon fringe pattern. Fluorescence dynamics were measured using a transient recorder connected to a computer which carried out add-to-memory averaging.

Samples were prepared using $248 \mathrm{Cm}\left(\mathrm{t}_{1 / 2}=3.4 \times 10^{5}\right.$ y) by co-precipitation of $\mathrm{Cm}^{3}+$ and $\mathrm{Ce}^{3+}$ as fluorides, followed by heating in HF gas and then in excess $F_{2}$ gas. The resulting powders were sealed off in quartz tubes containing $\mathrm{He}$ gas to ensure good thermal contact. A portion of the $\mathrm{Cm} 0.001 \mathrm{Ce} 0.999 \mathrm{~F} 4$ sample underwent analysis by $\mathrm{x}$-ray powder diffraction to verify that the structure of the material was the same as that of $\mathrm{CeF}_{4}$. The stated stoichiometry of the samples is based on the concentrations of $\mathrm{Ce}^{3+}$ and $\mathrm{Cm}^{3+}$ in aqueous solution prior to precipitation.

\section{SPECTRAL CHARACTERISTICS}

The host material, $\mathrm{CeF}_{4}$, is isostructural with $\mathrm{UF}_{4}[12]$ and $\mathrm{BkF}_{4}[13]$. There are two different low symmetry sites, $\mathrm{C}_{2}$ and $\mathrm{C}_{\mathrm{S}}$, for $\mathrm{Ce}^{4+}$, each of which is surrounded by eight fluorine ions arranged in a slightly distorted antiprism configuration. Substitution for $\mathrm{Ce}^{4+}$ places $\mathrm{Cm}^{4+}$ on sites of different symmetry and non-equivalent local environments. Consequently, differences in energy level structure and dynamics are expected for $\mathrm{Cm}^{4+}$ on the two crystalographic sites.

High-resolution laser excitation was utilized to unravel the resulting complex multisite spectra and to investigate the dynamics of excited $\mathrm{f}$-states of $\mathrm{Cm}^{4+}$ on the non-equivalent sites, hereafter termed sites A and B. The observed excitation and emission spectra consisted of both purely electronic and vibronic bands for the non-equivalent ion sites. The peak intensity of the broad vibronic transitions generally were comparable to the narrow electronic zero-phonon lines within a multiplet. Figure 1 shows the observed energy level structure of $\mathrm{Cm}^{4}+$ in $\mathrm{CeF}_{4}$ at $4.2 \mathrm{~K}$. Each energy band begins, at lowest energy, with one or more narrow zero-phonon lines followed by broad vibronic lines extending over circa 500 $\mathrm{cm}^{-1}$. The observed energy levels for site $A$ and $B$ differ by less than $50 \mathrm{~cm}^{-1}$ for the lowest component 
in each multiplet. A detailed description and analysis of the $\mathrm{Cm}^{4+}$ spectra is being prepared for publication [14].

\section{FLUORESCENCE DYNAMICS}

Using selective laser excitation, we have measured the f-state fluorescence decays of a metastable $J=1$ state of $\mathrm{Cm}^{4+}$ in $\mathrm{CeF}_{4}$ (see Fig. 1). The pulsed dye laser beam energy was varied from 20 to 500 $\mu \mathrm{J} /$ pulse. Nonexponential fluorescence decays were observed in both the $0.1 \%$ and $5 \% \mathrm{Cm}^{4+}$ samples at all laser powers and from $4.2 \mathrm{~K}$ to $300 \mathrm{~K}$. In the $5 \% \mathrm{Cm}^{4+}$ sample, the fluorescence decay was very rapid immediately after the laser pulse and became single exponential at long decay times. The nonexponential character of the observed fluorescence decays forces consideration of processes such as exciton-excition annihilation and cross relaxation.

The observed emission spectra indicate that the resonance transition is more than 100 -fold weaker than transitions from the emitting state to the lower-lying $J=1,2,3$ and 4 multiplets (see Fig. 1). The probability of donor-donor resonant energy transfer via dipole-dipole interaction is therefore small. On the other hand, cross relaxation may be very efficient because of comparatively high optical transition probabilities and small energy mismatch. There are seven final state combinations to lower observed levels that have energy mis-matches from 1 to $19 \mathrm{~cm}^{-1}$, given a pair of $\mathrm{Cm}^{4+}$ ions, each of the same site symmetery, with one ion initially in the emitting $\mathrm{J}=1$ state. Additional combinations may occur which involve components of the unobserved $\mathrm{J}=0,5$, and 6 states.

Since these cross-relaxation processes quench the emitting state, the intrinsic $\mathrm{Cm}^{4+}$ ions act as both donors and acceptors. Therefore, in the case of negligible resonant donor-donor transfer, the dynamics of the excited state can be described by an irreversible energy transfer model which gives a simple form for the fluorescence decay in a dilute system. With the assumption of dipole-dipole interaction, fluorescence intensity, $\mathrm{I}(\mathrm{t})$, decays as

$$
I(t)=I(0) \exp \left[-k 0 t-k 1 t^{1 / 2}\right]
$$


where $I(0)$ is the intensity at time $t=0, k_{0}$ is the reciprocal lifetime of the emission state, and $k_{1}$ is the integrated rate of energy transfer from donor to acceptor[2]. The observed fluorescence decays from the $0.1 \% \mathrm{Cm}^{4+}$ sample at low excitation energy are well-fit by Eq. 1 . The fit values of $\mathrm{k}_{0}$ and $\mathrm{k}_{1}$ are $6 \mathrm{x}$ $10^{3} \mathrm{~s}^{-1}$ and $5 \times 10^{1} \mathrm{~s}^{-1} / 2$, respectively. Figure $2 \mathrm{a}$ shows a typical fluorescence decay curve following excitation of $\mathrm{Cm}^{4+}$ in site $\mathrm{A}$ in the $0.1 \% \mathrm{Cm}^{4+}$ sample at $4.2 \mathrm{~K}$ while monitoring the lowest energy component of the emitting $\mathrm{J}=1$ multiplet to the lowest energy component of the lower-lying $\mathrm{J}=1$ multiplet was monitored (see Fig. 1).

The fluorescence decay from the $0.1 \% \mathrm{Cm}^{4+}$ sample exhibited an unusual excitation energy dependence in that the observed fluorescence decay became slower as the excited ion density was increased either by use of higher laser power or by shifting the laser frequency to a stronger absorption feature of the emitting $J=1$ state. These observations are explicable in terms of an exciton-exciton annihilation model described below, assuming the excitation intensity was sufficient to give rise to significant ground state depletion. The excited state population was found to increase supralinearly with increasing laser power based on the observed emission intensity at shortest times, an effect attributable to a two-photon process[15].

As the fraction of $\mathrm{Cm}^{4+}$ in the excited state increases, the ground state population of $\mathrm{Cm}^{4+}$ proportionately decreases which reduces the probability of cross relaxation in the sense of Eq. 1 . At sufficiently high fractional population of the excited state, cross relaxation involving two $\mathrm{Cm}^{4+}$ ions, both initially excited, must be taken into account. This exciton-exciton annihilation, resulting in upconversion, is most likely to occur at high excitation power[7-9], particularly in the $5 \% \mathrm{Cm}^{4+}$ sample. Indeed, the observation of efficient two-photon excitation[15] suggests that the up-conversion induced by exciton-exciton annihilation might be significant. Under these circumstances, exciton-exciton annihilation may become the predominant mechanism responsible for nonexponential decays.

Such exciton-exciton annihilation results in creation of a $\mathrm{Cm}^{4+}$ ion in a substantially higher excited state while the energy of the other $\mathrm{Cm}^{4+}$ ion lies below the emitting level. Due to a rapidly increasing 
density of states and smaller energy gaps with increasing energy, the up-converted ion would be expected to undergo rapid non-radiative decay back down to the emitting state. With an exciton-exciton annihilation model[7-9] which assumes all ions on a given site have equal probability of excitation, and taking into account emission from trap or defect site $\mathrm{Cm}^{4+}$, one can describe the excited state dynamics by the following coupled rate equations:

$$
\begin{aligned}
& \frac{d n_{1}}{d t}=-\left(k_{0}+k_{1}\right) n_{1}+k_{2} n_{1} 2 \\
& \frac{d n_{2}}{d t}=\gamma k_{1} n_{1}-k_{3} n_{2}
\end{aligned}
$$

The dynamics of excited state intrinsic $\mathrm{Cm}^{4+}$ are described by Eq. 2a, where $\mathrm{n} 1$ is the number density of excited ions, $\mathrm{k}_{0}$ is the purely radiative decay rate, $\mathrm{k}_{1}$ is the non-radiative decay rate (including energy transfer to trap sites), and $k_{2}$ is the coefficient of exciton-exciton annihilation. The dynamics of defect or trap site $\mathrm{Cm}^{4+}$ are described by Eq. $2 \mathrm{~b}$, where $\mathrm{n}_{2}$ is the excited state density of such sites, $\gamma$ is the fraction of intrinsic $\mathrm{Cm}^{4+}$ ions undergoing non-radiative decay which populate trap site $\mathrm{Cm}^{4+}$ whose emission overlap that of intrinsic $\mathrm{Cm}^{4+}$, and $\mathrm{k} 3$ is the sum of first order radiative and non-radiative decay rates of trap site $\mathrm{Cm}^{4+}$.

Eq. 2a alone was found to provide a good fit for the observed decays from $0.1 \% \mathrm{Cm}^{4+}$ at high excitation intensity. The observed decays from $5 \% \mathrm{Cm}^{4+}$ could not be fit by $\mathrm{Eq}$. $2 \mathrm{a}$ alone, due to an overlap between emission from intrinsic $\mathrm{Cm}^{4+}$ and defect or trap site $\mathrm{Cm}^{4+}$. Non-intrinsic site absorption features were found to be broad and to overlap the narrow zero-phonon lines of intrinsic site $\mathrm{Cm}^{4+}$ based on comparison with the $0.1 \% \mathrm{Cm}^{4+}$ spectra. Clear evidence of spectral migration from high to low frequencies of the defect-site emission was observed in the time resolved emission spectra after initial excitation on a major site[15]. 
The defect-site emission contribution to the observed decay curve from the $5 \% \mathrm{Cm}^{4+}$ sample at $4.2 \mathrm{~K}$ was accounted for by carrying out a non-linear least squares fitting of the integral form of Eqs. 2a and $2 b$. Figure $2 b$ shows the observed and resulting calculated decay curves for the same transition monitored in Figure 2a. From the fit (and assuming $k_{0}$ is $6 \times 10^{3} \mathrm{~s}^{-1}$ as found for $0.1 \% \mathrm{Cm}^{4+}$ ), we obtained $\mathrm{k}_{1}=3 \times 10^{4} \mathrm{~s}^{-1}, \mathrm{k}_{2}$ is of order $10^{-12} \mathrm{~cm}^{3} \mathrm{~s}^{-1}, \gamma$ is of order 0.1 , and $\mathrm{k}_{3}=8 \times 10^{3} \mathrm{~s}^{-1}$. The fit values are insensitive to variation of $\gamma$ by a factor of 2 . Determination of $k_{2}$ requires knowledge of the actual number density of excited states. Numerical integration of Eqs. $2 a$ and $2 b$ with trial values of $n 1$ at time 0 resulted in good agreement with the observed decay for $k_{2}$ of order $10-12 \mathrm{~cm}^{3} \mathrm{~s}^{-1}$, which is close to the exciton-exciton annihilation rate reported for $\mathrm{MnF}_{2}[7]$, and $\mathrm{n}_{1}$ at time 0 of order of 0.1 of the number density of $\mathrm{Cm}^{4+}$ in the $5 \%$ sample. Assuming $\mathrm{k} 2$ values in the $10^{-15}$ to $10^{-14} \mathrm{~cm}^{3} \mathrm{~s}^{-1}$ range (i.e., similar to that found for $\mathrm{TbPO}_{4}[8]$ or $\mathrm{TbF}_{3}[9]$ ) required a value for $\mathrm{n}_{1}$ over an order of magnitude higher than the total number density of $\mathrm{Cm}^{4+}$ in the sample.

\section{CONCLUSIONS}

The excited state dynamics of $\mathrm{Cm}^{4+}$ in $\mathrm{CeF}_{4}$ are complex even at $0.1 \% \mathrm{Cm}^{4+}$. The observed nonexponential fluorescence decays, which depend on actinide ion concentration, excitation intensity and excitation frequency, have been analyzed based on the mechanisms of cross relaxation and excitonexciton annihilation. In comparison with $3+$ lanthanide compounds, these results indicate that such energy transfer mechanisms are much stronger in 4+ actinide materials, an effect attributed to larger ionion interaction and higher density of states.

\section{ACKNOWLEDGEMENTS}

This work was performed under the auspices of the Office of Basic Energy Sciences, Division of Chemical Sciences, U. S. Department of Energy, under contract number W-31-109-ENG-38. We thank C. W. Williams for preparing and characterizing the samples, W. T. Carnall for helpful discussions concerning 4+ actinide ion energy level structure, and the transplutonium element production facilities at Oak Ridge National Laboratory for supplying the $248 \mathrm{Cm}$ used in this work. 


\section{REFERENCES}

1. W. M. Yen, in: Spectroscopy of Solids Containing Rare Earth Ions, eds. A. A. Kaplyanskii and R. M. Marcfarlane, (North-Holland, Amsterdam, 1987), p. 185.

2. T. T. Basiev, V. A. Malyshev and A. K. Przhevuskii, ibid., p. 275.

3. Y. K. Voron'ko, T. G. Mamedov, V. V. Osiko, A. M. Prokhorov, V. P. Sakun, and I. A. Shcherbakov, Sov. Phys. JETP, 44 (1976) 251.

4. J. Hegarty, D. L. Huber, W. M. Yen, Phys. Rev. B23 (1981) 6271 ; B25 (1982) 5638 .

5. D. L. Huber, D. S. Hamilton, B. Barnett, Phys. Rev. B16 (1977) 4642.

6. M. J. Weber, Phys. Rev. B8 (1973) 2124.

7. B. A. Wilson, J. Hegarty, and W. M. Yen, Phys. Rev. Lett., 41 (1978) 268.

8. P. C. Diggle, K. A. Gehring, and R. M. MacFarlane, Sol. St. Commun., 18 (1976) 391.

9. M. F. Joubert, B. Jacquir, and R. Moncorge, Phys. Rev. B28 (1983) 3725.

10. W. T. Carnall and H. M. Crosswhite, Optical Spectra and Electronic Structure of Actinide Ions in Compounds and Solution, Argonne National Laboratory Report ANL-84-90, (August 1985).

11. J. C. Krupa, Inorg. Chim. Acta, 139 (1987) 233.

12. A. C. Larson, R. B. Roof, D. T. Cromer, Acta Cryst. 17 (1964) 555. 
13. D. D. Ensor, J. R. Peterson, R. G. Haire and J. P. Young, J. Inorg. Nucl. Chem., 43 (1981) 1001.

14. G. K. Liu, J. V. Beitz, W. T. Carnall, G. L. Goodman, C. W. Williams, to be published.

15. G. K. Liu and J. V. Beitz, to be published. 


\section{Figure Captions}

Figure 1.

The observed energy level structure of $\mathrm{Cm}^{4+}$ is shown at the left. The energy extent of each observed multiplet (including vibronic bands) is shaded with diagonal lines and its probable J value is shown[14]. All observed fluorescence comes from the second excited $\mathbf{J}=1$ state as shown by the downward pointing arrows which are the highest energies of the observed fluorescence bands at $4.2 \mathrm{~K}$.

Figure 2.

Observed fluorescence intensity decay data $\left(\bullet\right.$, every 10 th value plotted), from $\mathrm{Cm}^{4+}$ at 4.2 $\mathrm{K}$ in $\mathrm{Cm} 0.001 \mathrm{Ce} 0.999 \mathrm{~F}_{4}$ at low excitation intensity (labelled a), and Cm0.05Ce0.95F4 (labelled b). Curve a, (solid line) results from fitting the observed data using a cross-relaxation model (Eq. 1) while curve b (solid line) corresponds to an exciton-exciton annihilation model (Eqs. 2a and 2b). 


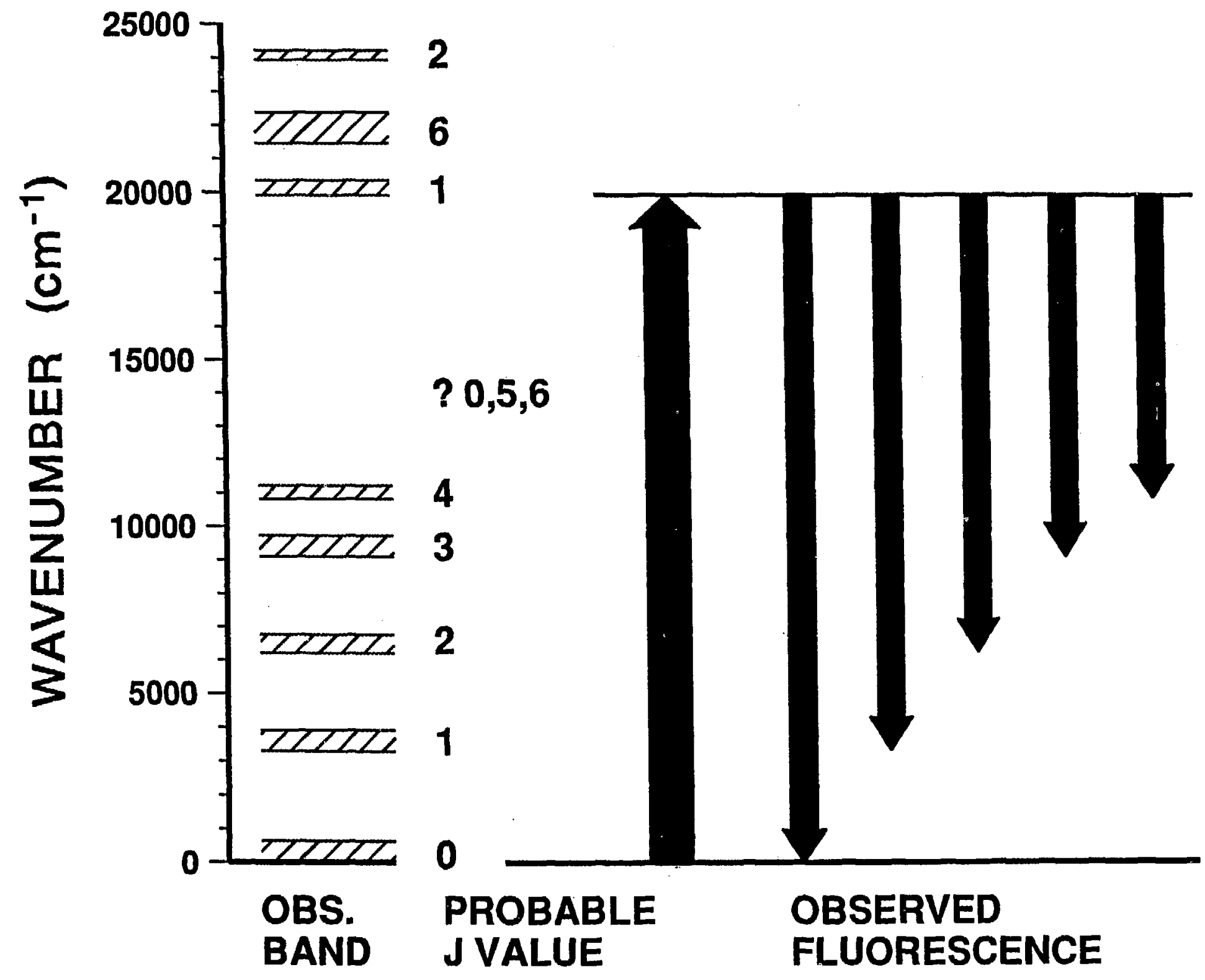


FLUOR. INTENSITY ( arb. unit )

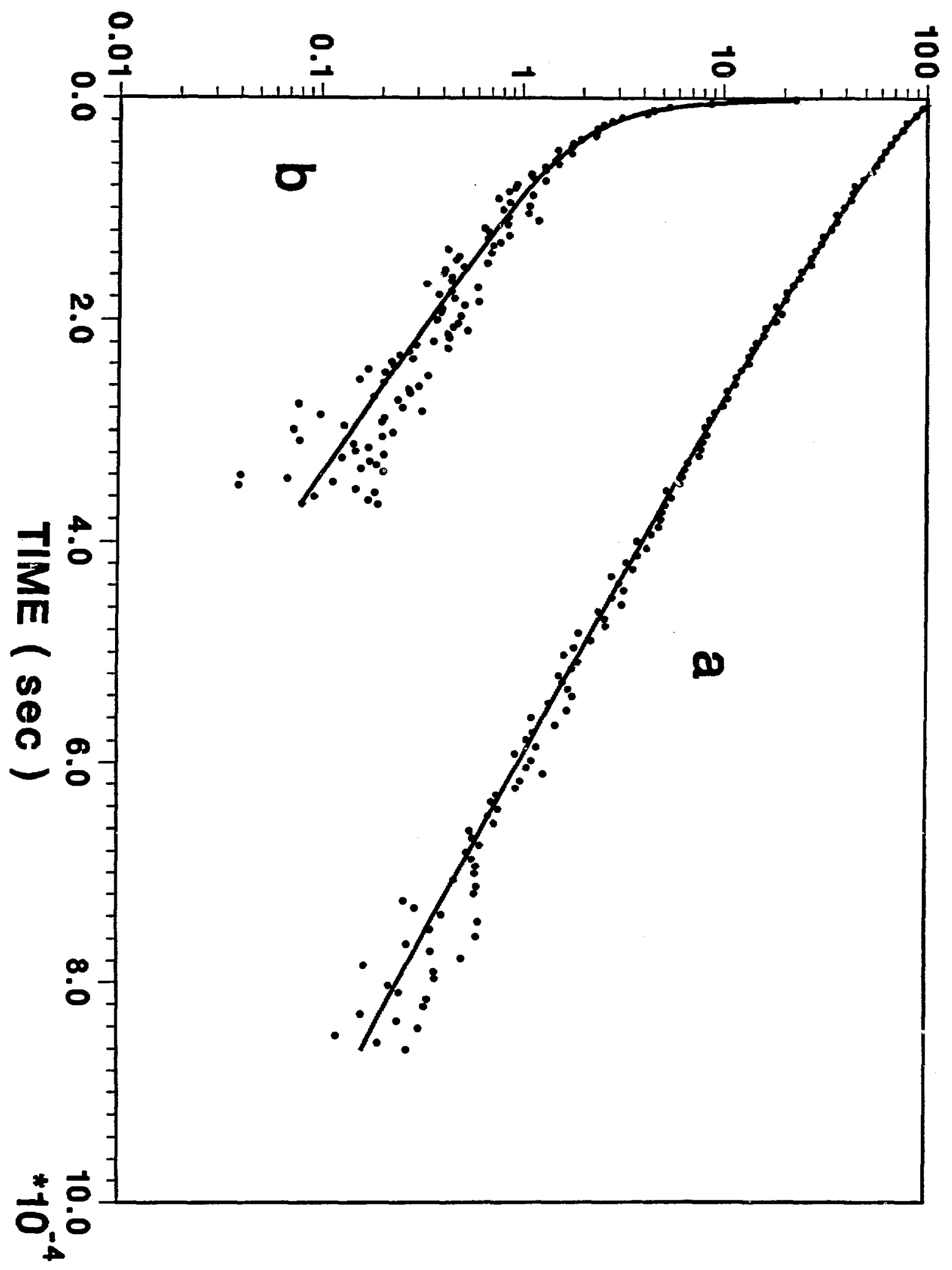

